\title{
MEASUREMENT OF SOLID SLURRY FLOW VIA CORRELATION OF ELECTROMAGNETIC FLOW METER, ELECTRICAL RESISTANCE TOMOGRAPHY AND MECHANISTIC MODELLING*
}

\author{
XU Jing-yu, WU Ying-xiang, ZHENG Zhi-chu \\ Institute of Mechanics, Chinese Academy of Sciences, Beijing 100190, China, E-mail: xujingyu@imech.ac.cn \\ WANG M., MUNIR B., OLUWADAREY H. I., SCHLABERG H. I., WILLIAMS R. A. \\ School of Process, Environmental and Materials Engineering, University of Leeds, Leeds, UK
}

(Received April 15, 2009, Revised May 25, 2009)

\begin{abstract}
The study presented here was carried out to obtain the actual solids flow rate by the combination of electrical resistance tomography and electromagnetic flow meter. A new in-situ measurement method based on measurements of the Electromagnetic Flow Meters (EFM) and Electrical Resistance Tomography (ERT) to study the flow rates of individual phases in a vertical flow was proposed. The study was based on laboratory experiments that were carried out with a $50 \mathrm{~mm}$ vertical flow rig for a number of sand concentrations and different mixture velocities. A range of sand slurries with median particle size from $212 \mu \mathrm{m}$ to $355 \mu \mathrm{m}$ was tested. The solid concentration by volume covered was $5 \%$ and $15 \%$, and the corresponding density of $5 \%$ was $1078 \mathrm{~kg} / \mathrm{m}^{3}$ and of $15 \%$ was $1238 \mathrm{~kg} / \mathrm{m}^{3}$. The flow velocity was between $1.5 \mathrm{~m} / \mathrm{s}$ and $3.0 \mathrm{~m} / \mathrm{s}$. A total of 6 experimental tests were conducted. The equivalent liquid model was adopted to validate in-situ volumetric solids fraction and calculate the slip velocity. The results show that the ERT technique can be used in conjunction with an electromagnetic flow meter as a way of measurement of slurry flow rate in a vertical pipe flow. However it should be emphasized that the EFM results must be treated with reservation when the flow pattern at the EFM mounting position is a non-homogenous flow. The flow rate obtained by the EFM should be corrected considering the slip velocity and the flow pattern.
\end{abstract}

Key words: slurry measurement, Electrical Resistance Tomography (ERT), Electromagnetic Flow Meters (EFM), in-situ mean volumetric fraction, slip velocity

\section{Introduction}

Slurry is an essential mixture of solid and liquid, and its physical characteristics are dependent on many factors such as the size and concentration distributions of solids in the liquid phase, size of the conduit, level of turbulence, temperature, and absolute (or apparent) viscosity of the carrier. The transport of solid-liquid slurries over short and medium distances via pipelines is very important in many industrial applications ${ }^{[1,2]}$.

* Project supported by the China-UK joint project of a study of multi-phase flow meter on EIT and CTA Techniques: Royal Society (Grant No. 15933).

Biography: Xu Jing-yu (1975-), Male, Ph. D.
Local solid hold-up is one of the most important hydrodynamic characteristics, which is needed for the design, analysis and performance estimation of liquid-solid two-phase flow and pipeline transportation systems.

Electromagnetic Flow Meters (EFM) have been successfully applied to measure mean velocities of single-phase liquid in industry. Continuous efforts have been made to determine the characteristics of two-phase flow using electromagnetic flow meters, since such meters do not introduce a pressure drop and can provide a fast response to changes in the flow. Thus, there are many potential applications for electromagnetic flow meters in two-phase flows. However, due to the complexity of multiphase flow in 
solid slurry transportation, it is difficult to accurately measure solid concentration and flow rate using a conventional electromagnetic flow meter alone. Normally, a secondary sensor, e.g., the gamma-ray density meter, has to be employed.

Electrical Resistance Tomography (ERT) is a measurement technique for non-intrusively mapping electrical conductivity distributions in process vessels and multiphase pipelines and has been used successfully in predicting the solid concentration, disperse phase velocity and flow regimes in both vertical and horizontal flows ${ }^{[3-7]}$. The ERT system operates by injecting a low current (typical $15 \mathrm{~mA}$ ) between adjacent pairs of neighbouring boundary electrodes, where the potential difference is measured by the remaining electrodes in some pre-determined fashion. The method used is dependent on the resources available and the computational time required in performing each calculation. The value of the in-situ phase fraction at a given location in the pipe can be related to the local mixture conductivity using relationships derived by Maxwell. However, the ERT is unable to measure the flow rate of the continuous phase and in its current form has difficulties in presenting an absolute value. In the present work, a new in-situ measurement method based on measurements with the EFM and ERT to study the flow rates of individual phases in a vertical flow is proposed and the correlation of EFM-ERT measurements together with mechanistic modelling is studied.

\section{Theoretical considerations}

\subsection{The slip velocity}

Slip is a phenomenon that usually occurs in a multi-phase flow. For a liquid-solid two-phase flow, the liquid phase moves much faster than the solid, except in a downward flow. The difference in the in-situ average velocities between the liquid and solid phases will result in a very important phenomenon, the "slip" of one phase relative to the other, or the "hold-up" of one phase relative to the other. This makes the in-situ volume fractions different from the solid loading volume fractions. It is of importance to study this phenomenon in detail in order to obtain an accurate in-situ fraction. Therefore, the present work will use different models to study the influence of the slip velocity on the slurry measurement. The first of these models is the hindered settling velocity, proposed by Richardson and Zaki, which can provide estimates for individual grains of sand. The hindered settling velocity, $v_{T}$, can be estimated by:

$$
v_{T}=v_{T}^{\prime}\left(1-\varepsilon_{S D}\right)^{n^{\prime}}
$$

where $\varepsilon_{S D}$ is the delivered volumetric solids fraction, the index, $n^{\prime}$, a function of the particle's Reynolds number, depends on the dimensionless particle diameter, which is $n^{\prime}=4.6$ for particles settling in the range of Stokes' law and $n^{\prime}=2.4$ in the range of Newton's law, respectively, $v_{T}^{\prime}$ is the terminal settling velocity obtained by Stokes' law and Newton's law.

When the Reynolds number is less than 0.1 in the Stokes region, the terminal settling velocity can be expressed by

$$
v_{T}^{\prime}=\frac{g d^{2}\left(\rho_{S}-\rho_{L}\right)}{18 \mu_{L}}
$$

where $\rho_{L}$ is the fluid density, $\mu_{L}$ is the fluid viscosity, $g$ is the acceleration due to gravity, $d$ is the particle diameter, and $\rho_{S}$ is the particle density.

When the Reynolds number is between 750 and $3 \times 10^{5}$, the drag coefficient is nearly constant with a value of 0.44 in what is known as the Newton's law region. The terminal settling velocity is then:

$$
v_{T}^{\prime}=1.73 \sqrt{\frac{g d\left(\rho_{S}-\rho_{L}\right)}{\rho_{L}}}
$$

\subsection{The in-situ volumetric solids fraction}

The frictional head loss can be described by the Equivalent Liquid Model (ELM) ${ }^{[8]}$. For vertical upward flow, the force balance can be given by

$\frac{\mathrm{d} p}{\mathrm{~d} x}=g \rho_{L} S_{M}+\frac{4 \tau_{M}}{D}$

where $\mathrm{d} p / \mathrm{d} x$ is the pressure gradient, $\tau_{M}$ is the shear stress, $D$ is the tube diameter, and $S_{M}$ is the in-situ relative density, given by

$S_{M}=1+\left(S_{S}-1\right) \varepsilon_{S}$

where $S_{S}=\rho_{S} / \rho_{L}$ and $\varepsilon_{S}$ represent the relative density of the solid and the in-situ volumetric solids fraction, respectively. According to this model the density of the mixture influences the liquid-like wall shear stress so that

$$
\tau_{M}=\tau_{L} \frac{\rho_{M}}{\rho_{L}}
$$


where $\rho_{M}=\rho_{S} \varepsilon_{S L}+\rho_{L}\left(1-\varepsilon_{S L}\right)$, in which $\varepsilon_{S L}$ is the solids loading volumetric fraction. The wall shear stress, $\tau_{L}$, is defined as

$$
\tau_{L}=\frac{\rho_{L} f_{L} V^{2}}{2}
$$

where $V$ is the mixture velocity, and friction factors, $f_{L}$, in a smooth pipe can be approximated by

$$
f_{L}=C_{L} \operatorname{Re}_{L}^{-n}
$$

where $C_{L}=0.079, n=0.25$, for turbulent flow, and $C_{L}=16, n=1$ for laminar flow. Substitution of Eq.(5) into Eq.(4) leads to

$$
\varepsilon_{S}=\frac{\frac{\left(\frac{\mathrm{d} p}{\mathrm{~d} x}-\frac{4 \tau_{M}}{D}\right)}{g \rho_{L}}-1}{S_{S}-1}
$$

where $\tau_{M}=\rho_{M} C_{L} R e_{L}^{-n} V^{2} / 2$. Equation (9) may be used to calculate approximately the in-situ mean volumetric solid fraction.

The ERT system was used to estimate the in-situ volumetric fraction based on the average of volumetric fractions of individual pixels which constitute the entire image. The simple calculation is given by

$\varepsilon_{S}=\frac{\sum_{i=1}^{i=m} \varepsilon_{S, i} A_{i}}{A_{\text {total }}}$

where $A_{i}, A_{\text {total }}$ and $\varepsilon_{S, I}$ are the area of pixel, the area of image (the cross-sectional area of pipe) and in-situ local volumetric disperse phase fraction, respectively.

\subsection{Electromagnetic flow meter}

The theory of the voltage-sensing flow meter was first developed by Shercliff. The weight function, which represents the degree of the contribution of the fluid velocity to the signal at the cross-section of a conduit, was proposed and computed for single-phase flow. For two-phase flow with non-uniform but isotropic conductivity, Bevir concluded that $\Delta U_{T P}$ can be expressed as

$$
\Delta U_{T P}=\frac{4 B Q_{L}}{\Lambda \pi d\left(1-\varepsilon_{S}\right)}=\frac{\Delta U_{S P}}{\Lambda\left(1-\varepsilon_{S}\right)}
$$

Here $Q_{L}$ is liquid flow rate and $\Delta U_{T P}$ is the potential difference between electrodes for two-phase flow and $\Delta U_{S P}$ for liquid flow alone (at the same flow rate $\left.Q_{L}\right) . \Lambda$ is a homogeneity factor based on the conductivity distribution over the cross section of the EFM sensor in accordance with the flow power law and asymmetric velocity profile.

Bernier and Brennen used an electromagnetic flow meter to measure a homogenous gas-liquid two-phase flow. They concluded that a homogenous flow would give rise to

$$
\Delta U_{T P}=\frac{4 B Q_{L}}{\pi d\left(1-\varepsilon_{S}\right)}=\frac{\Delta U_{S P}}{1-\varepsilon_{S}}
$$

They also showed that $\Delta U_{S P} / \Delta U_{T P}\left(1-\varepsilon_{S}\right)=1$ is also valid irrespective of flow regimes or the homogeneity of electrical conductivity.

The difference between the two approaches is obvious, i.e., whether the homogeneity of dispersive phase distribution (flow pattern) should be taken into consideration. Since ERT can present the dispersive phase in-situ distribution, we prefer to use Eq.(11) in the present work, which could be simplified as

$Q_{L}=\Lambda\left(1-\varepsilon_{s}\right) Q_{E F M}$

where $Q_{E F M}$ is the mixture flow rate obtained using EFM.

\section{Experimental setup and procedure}

A slurry flow loop with the inside diameter of 50 $\mathrm{mm}$ has been designed and built at the School of Process, Materials and Environmental Engineering at the University of Leeds as shown in Fig. $1^{[9,10]}$. The total PVC pipe work is $22 \mathrm{~m}$ in length, with a $3 \mathrm{~m}$ long vertical and two $5 \mathrm{~m}$ long horizontal testing sections in the loop. The loop consists of a 500-litres main mixing tank, where the solid and liquid are mixed homogeneously and introduced into the loop. A 250 litre measuring tank was employed to determine the delivered volumetric solid fraction at high flow velocities, as well as for verification of flow rate readings. A $15 \mathrm{~kW}$ Warman International 2/11/2 AH heavy-duty centrifugal pump was used to transfer the 


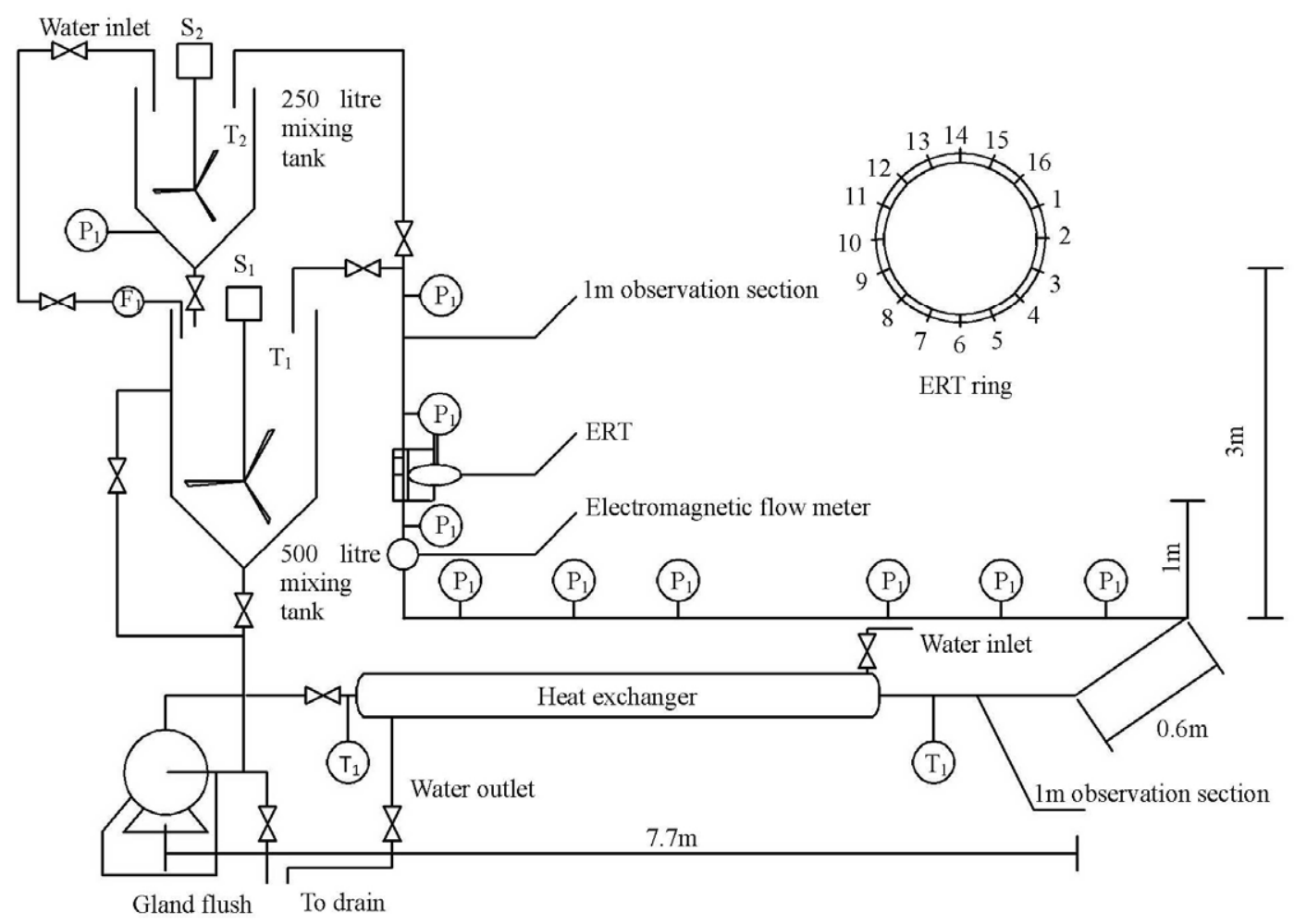

Fig.1 Schematic of test flow loop

slurry at velocities between $0.3 \mathrm{~m} / \mathrm{s}$ and $5 \mathrm{~m} / \mathrm{s}$. A frequency inverter was used for the control of the pump and hence the velocity and type of flow pattern that are generated. The flow rate of the solid and liquid mixture was measured by an EFM. The selected EFM was a Krohne Aquaflux unit because its body lining was manufactured to be resistant to the slurry material flowing through it. Therefore, an abrasive slurry could be investigated. Mounting the flow sensor on a vertical pipe allowed the measured velocity to be interpreted as the mixture velocity.

A range of sand slurries with median particle size from $212 \mu \mathrm{m}$ to $355 \mu \mathrm{m}$ was tested. The solid concentration by volume covered was $5 \%$ and $15 \%$, and the corresponding density of $5 \%$ is $1078 \mathrm{~kg} / \mathrm{m}^{3}$ and of $15 \%$ is $1238 \mathrm{~kg} / \mathrm{m}^{3}$. The flow velocity was between $1.5 \mathrm{~m} / \mathrm{s}$ and $3.0 \mathrm{~m} / \mathrm{s}$. A total of 6 experimental tests were conducted. The ERT results presented in this work were obtained from an ITS 2000 ERT system (Industrial Tomography Systems Ltd, Manchester, UK) in monitoring slurry transport in vertical pipes at several velocities. The ERT sensor was mounted in the working section at a distance of approximately $1.0 \mathrm{~m}$ from the tube bend. The dual-plane ERT sensor with two dummy rings was configured so that the axial separation of the image planes was $50 \mathrm{~mm}$. On each plane, sixteen stainless steel electrodes were mounted flush to the surface of the pipe at equal intervals. The electrodes were designed to have a length to width ratio of 3 , giving an electrode size of $18 \mathrm{~mm}$ by $6 \mathrm{~mm}$. The voltage potential differences for tomography images were collected based on the normal adjacent protocol, with a data collection speed of 5 frames per second for the dual planes, at an $\mathrm{AC}$ current injection frequency of $9600 \mathrm{~Hz}$ and a current value of $15 \mathrm{~mA}$. This produces 104 independent measurements for each tomographic image. The reconstruction of the image was carried out by the use of the Linear Back Protection (LBP) algorithm ${ }^{[11]}$. The volumetric solids fraction was determined from the Maxwell relationship ${ }^{[12]}$. In this work, before experiments we calibrated the ERT system and took the reference frame when the sensor was full of liquid only so that the reference measurement error could be controlled within $1 \%$.

\section{Results and discussion}

4.1 Measurement of in-situ volumetric solids fraction with ERT

For each experiment, 350 conductivity images were successively collected over approximately $5 \mathrm{~min}$, for all loading volumetric fraction. These images were averaged and merged and then the volumetric fraction profile was obtained using the Maxwell relationship. The results are presented in Fig.2. The solid curves represent theoretical results predicted by Eq.(9) and the points indicate the measured values using the ERT system. It is shown that the ERT system gives reasonable estimates of the mean volumetric fraction 
in the vertical flow. It also demonstrates that the mean concentration in the test section might be higher than that of the loading sand concentration. It is also observed that the decrease of in-situ volumetric fraction as mean mixture velocity (as well as the slip velocity) increases in the solid-water two-phase upward flow. This phenomenon is well known and can be explained with the principle of mass conservation and flow continuity. Furthermore, the comparison with the results obtained by the Equivalent Liquid Model (ELM) showed that ERT system provided a reasonable estimation of the average volumetric fraction for the mixture flow of the vertical liquid-solid slurry flow.

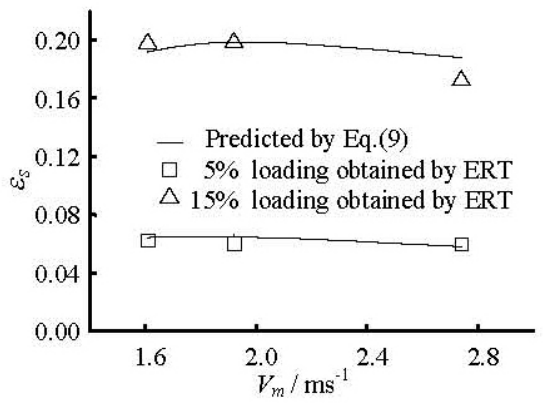

Fig.2 Comparison of volumetric solids fractions obtained with ERT system and ELM model

\subsection{Slurry flow rate measurement obtained by electromagnetic flow meter}

In order to investigate the effect of the mixture flow rate on the electromagnetic flow meter, we examine Eq.(13) using a loading volumetric sand fraction of 0.15. Two groups of data are presented in Fig.3. One set of data was obtained using the EFM readings corrected with a constant loading volumetric fraction, 0.15 , and the experimental measurement using a measurement tank, respectively. Another data set were obtained using the EFM readings corrected with the in-situ volumetric fractions measured with the ERT system at relevant mixture flow rates, respectively.

It can be seen from Fig. 3 that the data corrected by a constant volumetric fraction give a linear relationship between the mixture and water-phase flow rates. However a nonlinear correlation is presented from both the experimental measurement and the data with correction made with in-situ volumetric fractions. The gradient of the curve deceases gradually with increasing mixture flow rate. The difference may be caused by two factors: (1) the decrease of volumetric solids fraction due to the increase of slip velocity between solid phase and liquid phase, which are demonstrated in Fig.2, (2) the flow pattern trends to non-homogenous flow when slurry flow rate is increased, namely the homogeneity factor, $\Lambda$, may not be constant for a non-homogeneous system. The EFM readings corrected with in-situ volumetric fraction has a consistent tendency similar to that obtained by the experimental method.

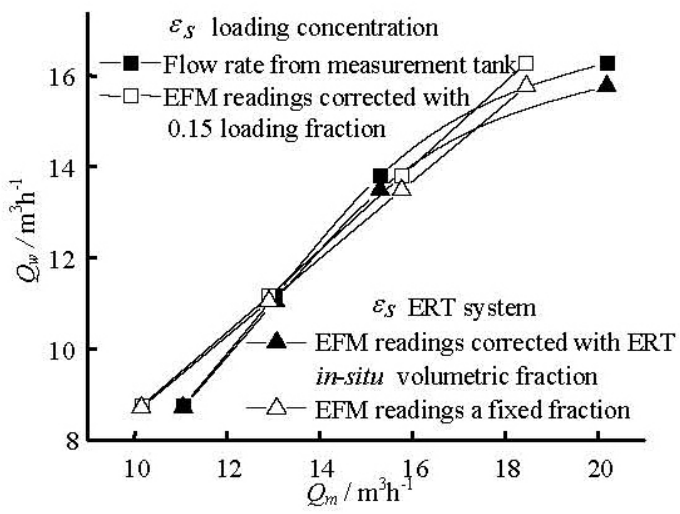

Fig.3 Flow rates obtained by EFM, EFM corrected with in-situ volumetric fraction and experimental measurements at a loading volumetric fraction of 0.15

\subsection{In-situ volumetric solids faction and velocity obtained by four different methods}

For studing the slip velocity $v_{T}$ in solid-liquid vertical flow, four different methods were attempted. If $\varepsilon_{S D}$ is defined as the delivered volumetric solids fraction, then we can obtain from continuity equation:

$v_{T}=V_{M} \frac{\varepsilon_{S}-\varepsilon_{S D}}{\varepsilon_{S}}$

$v_{T}$ plays a very important role in vertical hoisting. Due to the different flow patterns in horizontal and vertical pipes under the same entry conditions, the delivered volumetric solids fractions are different in different pipes, especially for the experiment loop of the present work. The solid and liquid are mixed homogeneously and first introduced into the horizontal pipe and then flows into the vertical pipe section. Thus the delivered volumetric solids fraction $\varepsilon_{S D}$ in the vertical pipe would be re-measured with $\mathrm{T}_{2}$ as is shown in Fig.1. Table 1 shows the results calculated with different models.

It is seen from Table 1 that Stokes' law gives the lowest $\varepsilon_{S}$ and $v_{T}$ values. It should be emphasized that in practice the Stokes and Newton models were based on the properties of single particles, so they only provide a rough estimate of the expected in-situ volumetric solids fraction. Furthermore, due to the particle size, velocity and density of the solid phase there will be segregation at the bottom of the vertical 
pipe. The two models have not taken into account the interaction between different sized particles, either. Thus it would be better that the results of ELM are used to validate the ERT system. It can be seen from Table 1 that the slip velocity in the test section reaches to $0.52 \mathrm{~m} / \mathrm{s}$ so that we should consider the effects of the slip velocity on slurry measurement. This aspect of the work will be conducted further to correct the measurement results of EFM in the future.

Table 1 A comparison of the mean in-situ volumetric solids fraction obtained and the slip velocity. $v_{T}$ by four methods (The mixture velocity, $V_{M}$, is $1.61 \mathrm{~m} / \mathrm{s})$. The arrow shows the derivation direction

$15 \%$ loading sand volume $\left(\varepsilon_{S D}=0.134\right)$

Methods

$\varepsilon_{S} \quad v_{T}$

$\begin{array}{ccc}\begin{array}{c}\text { Stokes' law } \\ \left(n^{\prime}=4.6\right)\end{array} & 0.138 & 0.051 \\ \begin{array}{c}\text { Newton' law } \\ \left(n^{\prime}=2.4\right)\end{array} & 0.140 & 0.073 \\ \text { ERT system } & 0.198 & 0.520 \\ \text { ELM } & 0.192 & 0.486\end{array}$

\subsection{Discussions}

By analyzing the results obtained above, for the measurement of flow rate it should be noted that the mounting position of the EFM instrument would have an important effect on results given by the EFM. In this study the EFM was mounted in the working section at a distance of about $0.1 \mathrm{~m}$ from the tube bend. As the mixture flows through a pipe bend this will result in an accumulation of particles at the bottom so that the accuracy of measurement is affected and there will be a distortion of velocity distribution in the pipe cross-section. In future work the EFM should be installed at a position where the vertical slurry flow is developed. Otherwise the EFM measurement has to be revised due to the influence of non-homogenous flow.

For the measurement of the disperse phase, a new in-situ measurement method based on ERT in a vertical flow is proposed. The results were checked by the ELM. Figure 4 shows that the axial solids volume fraction distribution obtained with the ERT system at various mixture velocities at the loading solid concentration of $5 \%$ and $15 \%$. It can be seen that there is an accumulation of particles at the outer wall of the pipe. Due to the ERT sensor being mounted in the working section at a distance of approximately $1.0 \mathrm{~m}$ from the tube bend and solid particle inertia, the mixture flow through a pipe bend will result in an accumulation of particles at the bottom and outer wall of the bend. In the connecting vertical pipe, the accumulation will disintegrate due to the secondary flow induced by the bend and due to flow turbulence. Similar results were also found by Huber and Sommerfeld in a gas-solid two-phase flow ${ }^{[13]}$. The results further prove the flow pattern of nonhomogenous flow in the test section showing that inevitable errors exist for the measurement of a non-homogenous two-phase flow using the EFM. Furthermore for non-homogenous flow the slip velocity has to be considered to correct the results of EFM, and the ELM was used to calculate the slip velocity and validate the ERT system.

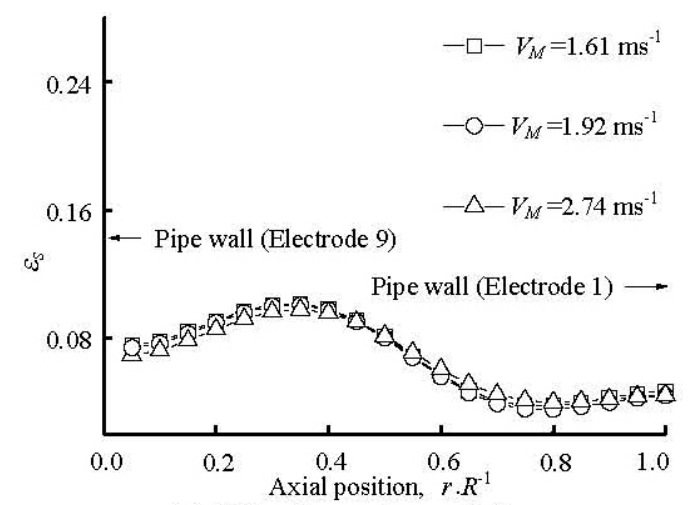

(a) $5 \%$ loading sand concentration

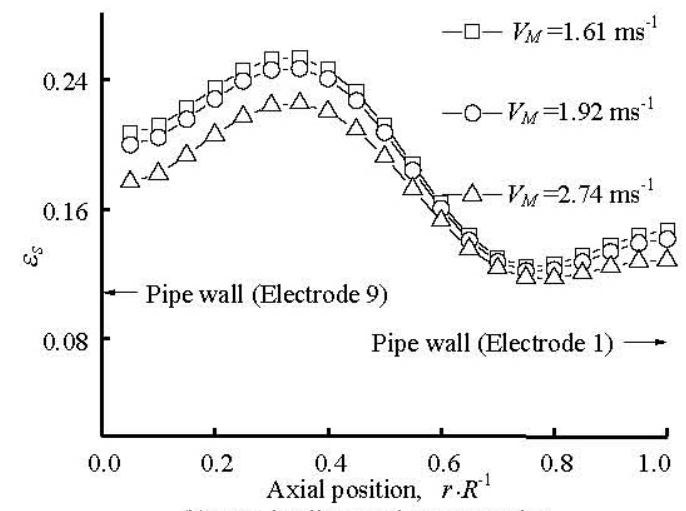

(b) $15 \%$ loading sand concentration

Fig.4 Axial solids volume fraction distribution obtained with ERT system

\section{Conclusion}

To understand the performance of a liquid-solid slurry flow in a vertical pipe and measure the individual phase flow rate, the present study has been carried out experimentally and theoretically to investigate the slurry vertical flow with the EFM and ERT techniques. A series of experiments have been 
conducted. As has been shown above, in the present work the flow rates of the continuous phase and two-phase mixture are measured using the EFM, the volumetric disperse phase fraction is obtained with an ERT system, and the slip velocity can be predicted with the ELM in a vertical pipe transporting slurry. The method suggested in this work can be used in the field of liquid-solid two-phase flow when the solid phase is as disperse and the fluid as continuous. However, it should be emphasized that the EFM must be treated with reservations when the flow pattern at the EFM mounting point is non-homogeneous. The slip velocity and flow pattern have to be considered to correct the results given by the equivalent liquid model. The results have demonstrated that the ERT technique can provide in-situ volumetric fraction and therefore can be used in conjunction with an electromagnetic flow meter as a way of measuring slurry flow rate in a vertical flow.

\section{References}

[1] CHENG Rong-chao, WANG Rui-he. A three-segment hydraulic model for annular cuttings transport with foam in horizontal drilling $[\mathrm{J}]$. Journal of Hydrodynamics, Ser. B, 2008, 20(1): 67-73.

[2] ZENG Zhuo-xiong, PAN Yang and ZHONG Chun et al. A second-order moment turbulence model for power law fluid with particles[J]. Journal of Hydrodynamics, Ser. B, 2007, 19(5): 607-612.

[3] MCKEE S. L., WILliAMS R. A. and BOXMAN A. Development of solid-liquid mixing models using tomographic techniques[J]. Chem. Eng. J., 1995, 56(33): 101-107.

[4] WILliamS R. A., JIA X. and MCKEE S. L. Development of slurry mixing models using resistance tomography[J]. Powder Tech., 1996, 87(1): 21-27.
[5] MANN R., WANG M. Electrical process tomography: simple and inexpensive techniques for process imaging[J]. Meas. Contr., 1997, 30: 206-211.

[6] LUCAS G. P., CORY J. and WATERFALL R. et al. Measurement of the solids volume fraction and velocity distributions in solids-liquid flows using dualplane electrical resistance tomography[J]. Flow Meas. Inst., 1999, 10(4): 249-258.

[7] WANG M., YIN W. Measurement of the concentration and velocity distribution in miscible liquid mixing using electrical resistance tomography[J]. Trans. Inst. Chem. Eng., 2001, 79(A8): 883-886.

[8] MATOUSEK V. Pressure drops and flow patterns in sand-mixture pipes[J]. Exp. Therm. Fluid Sci., 2002, 26(6-7): 693-702.

[9] PACHOWKO A. D., WANG M. and POOLE C. et al. The use of electrical resistance tomography (ERT) to monitor flow patterns in horizontal slurry transport pipelines[C]. Proceedings for 3rd World Congress on Industrial Process Tomography. Banff, Canada, VCIPT, 2003, 305-311.

[10] PACHOWKO A. D. Design and modelling of a coarse particulate slurry handling system[D]. Ph. D. Thesis, Leeds, UK: University of Leeds, 2004.

[11] WANG M. Inverse solutions for electrical impedance tomography based on conjugate gradients methods[J]. Meas. Sci. Tech., 2002, 13(1): 101-117.

[12] DYAKOWSKI T., JEANMEURE L. F. C. and JAWORSKI A. J. Applications of electrical resistance tomography for gas-liquid and liquid-solids flows- A review[J]. Powder Technology, 2000, 112(1-2): 174-192.

[13] HUBER N., SOMMERFELD M. Characterization of the cross-sectional particle concentration distribution in pneumatic conveying systems[J]. Powder Technology, 1994, 79(3): 191-210. 